\title{
A new semidefinite programming hierarchy for cycles in binary matroids and cuts in graphs
}

\author{
João Gouveia • Monique Laurent • \\ Pablo A. Parrilo • Rekha Thomas
}

Received: 26 July 2009 / Accepted: 7 October 2010 / Published online: 28 October 2010

(C) The Author(s) 2010. This article is published with open access at Springerlink.com

\begin{abstract}
The theta bodies of a polynomial ideal are a series of semidefinite programming relaxations of the convex hull of the real variety of the ideal. In this paper we construct the theta bodies of the vanishing ideal of cycles in a binary matroid. Applied to cuts in graphs, this yields a new hierarchy of semidefinite programming relaxations of the cut polytope of the graph. If the binary matroid avoids certain minors we can
\end{abstract}

Gouveia, Parrilo and Thomas were partially supported by the NSF Focused Research Group grants DMS-0757371 and DMS-0757207. Gouveia was also partially supported by Fundação para a Ciência e Tecnologia and Thomas by the Robert R. and Elaine K. Phelps Endowment at the University of Washington.

J. Gouveia $(\varangle) \cdot$ R. Thomas

Department of Mathematics, University of Washington, Box 354350, Seattle, WA 98195, USA

e-mail: jgouveia@math.washington.edu

R. Thomas

e-mail: thomas@math.washington.edu

J. Gouveia

CMUC, Department of Mathematics, University of Coimbra, 3001-454 Coimbra, Portugal

M. Laurent

CWI, Science Park 123, 1098 XG Amsterdam, The Netherlands

M. Laurent

Department of Econometrics and Operations Research, Tilburg University, Tilburg, The Netherlands e-mail: monique@cwi.nl

P. A. Parrilo

Department of Electrical Engineering and Computer Science,

Laboratory for Information and Decision Systems, Massachusetts Institute of Technology,

77 Massachusetts Avenue, Cambridge, MA 02139-4307, USA

e-mail: parrilo@mit.edu 
characterize when the first theta body in the hierarchy equals the cycle polytope of the matroid. Specialized to cuts in graphs, this result solves a problem posed by Lovász.

Keywords Theta bodies · Binary matroid - Cycle ideal · Cuts · Cut polytope · Combinatorial moment matrices $\cdot$ Semidefinite relaxations $\cdot \mathrm{TH}_{1}$-exact

\section{Mathematics Subject Classification (2000) $\quad 12 \cdot 52 \cdot 90$}

\section{Introduction}

A central question in combinatorial optimization is to understand the polyhedral structure of the convex hull, $\operatorname{conv}(S)$, of a finite set $S \subseteq \mathbb{R}^{n}$. A typical instance is when $S$ is the set of incidence vectors of a finite set of objects over which one is interested to optimize; think for instance of the problem of finding a shortest tour, a maximum independent set, or a maximum cut in a graph. As for hard combinatorial optimization problems one cannot hope in general to be able to find the complete linear description of the polytope $\operatorname{conv}(S)$, the objective is then to find good and efficient approximations of this polytope. Such approximations could be polyhedra, obtained by considering classes of valid linear inequalities. In recent years more general convex semidefinite programming (SDP) relaxations have been considered, which sometimes yield much tighter approximations than those from LP methods. This was the case for instance for the approximation of stable sets and coloring in graphs via the theta number introduced by Lovász [18], and for the approximation of the max-cut problem by Goemans and Williamson [8]. See e.g. [17] for an overview. These results spurred intense research activity on constructing stronger SDP relaxations for combinatorial optimization problems (cf. [12,13, 17, 20,22,23]). In this paper we revisit the hierarchy of SDP relaxations proposed by Gouveia et al. [9] which was inspired by a question of Lovász [19]. To present it we need some definitions.

Let $I \subseteq \mathbb{R}[\mathbf{x}]$ be an ideal and $V_{\mathbb{R}}(I)=\left\{\mathbf{x} \in \mathbb{R}^{n} \mid f(\mathbf{x})=0 \forall f \in I\right\}$ be its real variety. Throughout $\mathbb{R}[\mathbf{x}]$ denotes the ring of multivariate polynomials in $n$ variables $\mathbf{x}=\left(x_{1}, \ldots, x_{n}\right)$ over $\mathbb{R}$ and $\mathbb{R}[\mathbf{x}]_{d}$ its subspace of polynomials of degree at most $d \in \mathbb{N}$. As the convex hull of $V_{\mathbb{R}}(I)$ is completely described by the (linear) polynomials $f \in \mathbb{R}[\mathbf{x}]_{1}$ that are non-negative on $V_{\mathbb{R}}(I)$, relaxations of $\operatorname{conv}\left(V_{\mathbb{R}}(I)\right)$ can be obtained by considering sufficient conditions for the non-negativity of linear polynomials on $V_{\mathbb{R}}(I)$.

A polynomial $f \in \mathbb{R}[\mathbf{x}]$ is said to be a sum of squares (sos, for short) if $f=\sum_{i=1}^{t} g_{i}^{2}$ for some polynomials $g_{i} \in \mathbb{R}[\mathbf{x}]$. Moreover, $f$ is said to be sos modulo the ideal $I$ if $f=\sum_{i=1}^{t} g_{i}^{2}+h$ for some polynomials $g_{i} \in \mathbb{R}[\mathbf{x}]$ and $h \in I$. In addition, if each $g_{i}$ has degree at most $k$, then we say that $f$ is $k$-sos modulo I. Obviously any polynomial which is $k$-sos modulo $I$ is non-negative over $V_{\mathbb{R}}(I)$. Following [9], for each $k \in \mathbb{N}$, define the set

$$
\mathrm{TH}_{k}(I):=\left\{\mathbf{x} \in \mathbb{R}^{n} \mid f(\mathbf{x}) \geq 0 \text { for all } f \in \mathbb{R}[\mathbf{x}]_{1} k \text {-sos modulo } I\right\}
$$

called the $k$-th theta body of the ideal $I$. Note that $\operatorname{TH}_{k}(I)$ is a (convex) relaxation of $\operatorname{conv}\left(V_{\mathbb{R}}(I)\right)$, with 


$$
\operatorname{conv}\left(V_{\mathbb{R}}(I)\right) \subseteq \mathrm{TH}_{k+1}(I) \subseteq \mathrm{TH}_{k}(I)
$$

The ideal $I$ is said to be $\mathrm{TH}_{k}$-exact if the equality $\overline{\operatorname{conv}\left(V_{\mathbb{R}}(I)\right)}=\mathrm{TH}_{k}(I)$ holds. The theta bodies $\mathrm{TH}_{k}(I)$ were introduced in [9], inspired by a question of Lovász [19, Problem 8.3] asking to characterize $\mathrm{TH}_{k}$-exact ideals, in particular when $k=1$.

This question of Lovász was motivated by the following result about stable sets in graphs: The stable set ideal of a graph $G=(V, E)$ is $\mathrm{TH}_{1}$-exact if and only if the graph $G$ is perfect. Recall that a subset of $V$ is stable in $G$ if it contains no edge. The stable set ideal of $G$ is the vanishing ideal of the $0 / 1$ characteristic vectors of the stable sets in $G$ and is generated by the binomials $x_{i}^{2}-x_{i}(i \in V)$ and $x_{i} x_{j}(\{i, j\} \in E)$ (cf. [19] for details).

For a graph $G$, let $I G$ be the vanishing ideal of the incidence vectors of cuts in $G$, and the cut polytope, $\operatorname{CUT}(G)$, be the convex hull of the incidence vectors of cuts in $G$. Following Problem 8.3, Problem 8.4 in [19] asks for a characterization of "cutperfect" graphs which are precisely those graphs $G$ for which $I G$ is $\mathrm{TH}_{1}$-exact. We answer this question (Corollary 4.12) by studying theta bodies in the more general setting of cycles in binary matroids. As an intermediate step we derive the theta bodies of $I G$ which give rise to a new hierarchy of semidefinite programming relaxations of $\operatorname{CUT}(G)$.

\section{Some notation}

Let $E$ be a finite set. For a subset $F \subseteq E$, let $\mathbf{1}^{F} \in\{0,1\}^{E}$ denote its $0 / 1$-incidence vector and $\chi^{F} \in\{ \pm 1\}^{E}$ its \pm 1 -incidence vector, defined by $\mathbf{1}_{e}^{F}=1, \chi_{e}^{F}=-1$ if $e \in F$ and $\mathbf{1}_{e}^{F}=0, \chi_{e}^{F}=1$ otherwise. Throughout $\mathbb{R} E:=\mathbb{R}\left[x_{e} \mid e \in E\right]$ denotes the polynomial ring with variables indexed by $E$. If $F \subseteq E$, we set $\mathbf{x}^{F}:=\prod_{e \in F} x_{e}$. For a symmetric matrix $X \in \mathbb{R}^{n \times n}, X \succeq 0$ means that $X$ is positive semidefinite, or equivalently, $\mathbf{u}^{T} X \mathbf{u} \geq 0$ for all $\mathbf{u} \in \mathbb{R}^{n}$.

\section{Contents of the paper}

Section 2 contains various preliminaries and some results of [9] needed in this paper. In Sect. 3 we introduce binary matroids, which provide the natural setting to present our results for cuts in graphs. A binary matroid is a pair $\mathcal{M}=(E, \mathcal{C})$ where $E$ is a finite set and $\mathcal{C}$ is a collection of subsets of $E$ (the cycles of $\mathcal{M}$ ) closed under taking symmetric differences; for instance, cuts (resp., cycles) in a graph form binary matroids. In Sect. 3.1 we present a generating set for the cycle ideal $I \mathcal{M}$ (i.e. the vanishing ideal of the incidence vectors of the cycles $C \in \mathcal{C}$ ) and a linear basis $\mathcal{B}$ of its quotient space $\mathbb{R} E / I \mathcal{M}$ (cf. Theorem 3.4). Using this, we can explicitly describe the series of theta bodies $\mathrm{TH}_{k}(I \mathcal{M})$ that approximate the cycle polytope $\mathrm{CYC}(\mathcal{M})$ (i.e. the convex hull of the incidence vectors of the cycles in $\mathcal{C}$ ). In Sect. 3.2, we specialize these results to cuts in a graph $G$ and show that $\mathcal{B}$ can then be indexed by $T$-joins of $G$. This enables a combinatorial description of the theta bodies $\operatorname{TH}_{k}(I G)$ that converge to the cut polytope $\operatorname{CUT}(G)$ of $G$. Section 3.3 compares the semidefinite relaxations $\operatorname{TH}_{k}(I G)$ 
to some known semidefinite relaxations of the cut polytope. In Sect. 3.4 the results from Sect. 3.1 are specialized to cycles in a graph. Section 3.5 contains a discussion about the complexity of constructing theta bodies. Section 4 studies the binary matroids $\mathcal{M}$ whose cycle ideal $I \mathcal{M}$ is $\mathrm{TH}_{1}$-exact (i.e., $\mathrm{TH}_{1}(I \mathcal{M})=\mathrm{CYC}(\mathcal{M})$ ). Theorem 4.6 characterizes the $\mathrm{TH}_{1}$-exact cycle ideals $I \mathcal{M}$ when $\mathcal{M}$ does not have the three special minors $F_{7}^{*}, R_{10}$ and $\mathcal{M}_{K_{5}}^{*}$. As an application, we obtain characterizations of $\mathrm{TH}_{1}$-exact graphic and cographic matroids, and the latter answers Problem 8.4 in [19]. The paper contains several examples of binary matroids for which we exhibit the least $k$ for which $I \mathcal{M}$ is $\mathrm{TH}_{k}$-exact. In Sect. 5 we do this computation for an infinite family of graphs; if $C_{n}$ is the circuit with $n$ edges, then the smallest $k$ for which $\operatorname{TH}_{k}\left(I C_{n}\right)=\operatorname{CUT}\left(C_{n}\right)$ is $k=\lceil n / 4\rceil$.

\section{Preliminaries}

\subsection{Ideals and combinatorial moment matrices}

Let $\mathbb{R}[\mathbf{x}]$ be the polynomial ring over $\mathbb{R}$ in the variables $\mathbf{x}=\left(x_{1}, \ldots, x_{n}\right)$. A non-empty subset $I \subseteq \mathbb{R}[\mathbf{x}]$ is an ideal if $I$ is closed under addition, and multiplication by elements of $\mathbb{R}[\mathbf{x}]$. The ideal generated by $\left\{f_{1}, \ldots, f_{s}\right\} \subseteq \mathbb{R}[\mathbf{x}]$ is the set $I=\left\{\sum_{i=1}^{s} h_{i} f_{i}\right.$ : $\left.h_{i} \in \mathbb{R}[\mathbf{x}]\right\}$, denoted as $I=\left(f_{1}, \ldots, f_{s}\right)$. For $S \subseteq \mathbb{R}^{n}$, the vanishing ideal of $S$ is $\mathcal{I}(S):=\{f \in \mathbb{R}[\mathbf{x}] \mid f(\mathbf{x})=0 \forall \mathbf{x} \in S\}$. For $W \subseteq[n], I_{W}:=I \cap \mathbb{R}\left[x_{i} \mid i \in W\right]$ is the elimination ideal of $I$ with respect to $W$.

An ideal $I \subseteq \mathbb{R}[\mathbf{x}]$ is said to be zero-dimensional if its (complex) variety:

$$
V_{\mathbb{C}}(I):=\left\{\mathbf{x} \in \mathbb{C}^{n} \mid f(x)=0 \forall f \in I\right\},
$$

is finite, $I$ is radical if $f^{m} \in I$ implies $f \in I$ for any $f \in \mathbb{R}[\mathbf{x}]$, and $I$ is real radical if $f^{2 m}+\sum_{i=1}^{t} g_{i}^{2} \in I$ implies $f \in I$ for all $f, g_{i} \in \mathbb{R}[\mathbf{x}]$. By the Real Nullstellensatz (cf. [3]), $I$ is real radical if and only if $I=\mathcal{I}\left(V_{\mathbb{R}}(I)\right.$ ). Therefore, $I$ is zero-dimensional and real radical if and only if $I=\mathcal{I}(S)$ for a finite set $S \subseteq \mathbb{R}^{n}$. If $I$ is real radical, and $\pi_{W}$ denotes the projection from $\mathbb{R}^{[n]}$ to $\mathbb{R}^{W}$, then the elimination ideal $I_{W}$ is the vanishing ideal of $\pi_{W}\left(V_{\mathbb{R}}(I)\right)$, and there is a simple relationship between the $k$-th theta body of $I$ and that of its elimination ideal $I_{W}$ :

$$
\pi_{W}\left(\mathrm{TH}_{k}(I)\right) \subseteq \mathrm{TH}_{k}\left(I_{W}\right)
$$

The quotient space $\mathbb{R}[\mathbf{x}] / I$ is a $\mathbb{R}$-vector space whose elements, called the cosets of $I$, are denoted as $f+I(f \in \mathbb{R}[\mathbf{x}])$. For $f, g \in \mathbb{R}[\mathbf{x}], f+I=g+I$ if and only if $f-g \in I$. The degree of $f+I$ is defined as the smallest possible degree of $g \in \mathbb{R}[\mathbf{x}]$ such that $f-g \in I$. The vector space $\mathbb{R}[\mathbf{x}] / I$ has finite dimension if and only if $I$ is zero-dimensional; moreover, $\left|V_{\mathbb{C}}(I)\right| \leq \operatorname{dim} \mathbb{R}[\mathbf{x}] / I$, with equality if and only if $I$ is radical.

Gouveia et al. [9] give a geometric characterization of zero-dimensional real radical ideals that are $\mathrm{TH}_{1}$-exact. 
Definition 2.1 For $k \in \mathbb{N}$, a finite set $S \subseteq \mathbb{R}^{n}$ is said to be $k$-level if $\mid\{f(\mathbf{x}) \mid \mathbf{x} \in$ $S\} \mid \leq k$ for all $f \in \mathbb{R}[\mathbf{x}]_{1}$ for which the linear inequality $f(\mathbf{x}) \geq 0$ induces a facet of the polytope $\operatorname{conv}(S)$.

Theorem 2.2 [9] Let $S \subseteq \mathbb{R}^{n}$ be a finite set. The ideal $\mathcal{I}(S)$ is $\mathrm{TH}_{1}$-exact (i.e., $\left.\operatorname{conv}(S)=\mathrm{TH}_{1}(\mathcal{I}(S))\right)$ if and only if $S$ is a 2-level set.

More generally, Gouveia et al. [9, Sect. 4] show the implication:

$$
S \text { is }(k+1) \text {-level } \Longrightarrow \mathcal{I}(S) \text { is } \mathrm{TH}_{k} \text {-exact; }
$$

the reverse implication however does not hold for $k \geq 2$ (see e.g. Remark 5.8 for a counterexample).

We now mention an alternative more explicit formulation for the theta body $\mathrm{TH}_{k}(I)$ of an ideal $I$ in terms of positive semidefinite combinatorial moment matrices. We first recall this class of matrices (introduced in [16]) which amounts to using the equations defining $I$ to reduce the number of variables. Let $\mathcal{B}=\left\{b_{0}+I, b_{1}+I, \ldots\right\}$ be a basis of $\mathbb{R}[\mathbf{x}] / I$ and, for $k \in \mathbb{N}$, let $\mathcal{B}_{k}:=\{b+I \in \mathcal{B} \mid \operatorname{deg}(b+I) \leq k\}$. Then any polynomial $f \in \mathbb{R}[\mathbf{x}]$ has a unique decomposition $f=\sum_{l \geq 0} \lambda_{l}^{(f)} b_{l}$ modulo $I$; we let $\lambda^{(f)}=\left(\lambda_{l}^{(f)}\right)_{l}$ denote the vector of coordinates of the coset $f+I$ in the basis $\mathcal{B}$ (which has only finitely many non-zero coordinates).

Definition 2.3 Let $\mathbf{y} \in \mathbb{R}^{\mathcal{B}}$. The combinatorial moment matrix $M_{\mathcal{B}}(\mathbf{y})$ is the (possibly infinite) matrix indexed by $\mathcal{B}$ whose $(i, j)$-th entry is

$$
\sum_{l \geq 0} \lambda_{l}^{\left(b_{i} b_{j}\right)} y_{l} .
$$

The $k$ th truncated combinatorial moment matrix $M_{\mathcal{B}_{k}}(\mathbf{y})$ is the principal submatrix of $M_{\mathcal{B}}(\mathbf{y})$ indexed by $\mathcal{B}_{k}$.

In other words, the matrix $M_{\mathcal{B}}(\mathbf{y})$ is obtained as follows. The coordinates $y_{l}$ 's correspond to the elements $b_{l}+I$ of $\mathcal{B}$; expand the product $b_{i} b_{j}$ in terms of the basis $\mathcal{B}$ as $b_{i} b_{j}=\sum_{l} \lambda_{l}^{\left(b_{i} b_{j}\right)} b_{l}$ modulo $I$; then the $\left(b_{i}, b_{j}\right)$-th entry of $M_{\mathcal{B}}(\mathbf{y})$ is its 'linearization': $\sum_{l} \lambda_{l}^{\left(b_{i} b_{j}\right)} y_{l}$.

To control which entries of $\mathbf{y}$ are involved in the truncated matrix $M_{\mathcal{B}_{k}}(\mathbf{y})$, it is useful to suitably choose the basis $\mathcal{B}$. Namely, we choose $\mathcal{B}$ satisfying the following property:

$$
\operatorname{deg}(f+I) \leq k \Longrightarrow f+I \in \operatorname{span}\left(\mathcal{B}_{k}\right) .
$$

This is true, for instance, when $\mathcal{B}$ is the set of standard monomials of a term order that respects degree. (See [4, Chapter 2] for these notions that come from Gröbner basis theory.) If $\mathcal{B}$ satisfies (4), then the entries of $M_{\mathcal{B}_{k}}(\mathbf{y})$ depend only on the entries of $\mathbf{y}$ indexed by $\mathcal{B}_{2 k}$. Moreover, Gouveia et al. [9] show that $\mathrm{TH}_{k}(I)$ can then be defined using the matrices $M_{\mathcal{B}_{k}}(\mathbf{y})$, up to closure and a technical condition on $\mathcal{B}$. This 
technical condition, which states that $\left\{1+I, x_{1}+I, \ldots, x_{n}+I\right\}$ is linearly independent in $\mathbb{R}[\mathbf{x}] / I$, is however quite mild since if there is a linear dependency then it can be used to eliminate variables.

Example 2.4 Consider the ideal $I=\left(x_{1}^{2} x_{2}-1\right) \subset \mathbb{R}\left[x_{1}, x_{2}\right]$. Note that $\mathcal{B}=$ $\bigcup_{k \in \mathbb{N}}\left\{x_{1}^{k}+I, x_{2}^{k}+I, x_{1} x_{2}^{k}+I\right\}$ is a monomial basis for $\mathbb{R}\left[x_{1}, x_{2}\right] / I$ satisfying (4) for which

$$
\mathcal{B}_{4}=\left\{1, x_{1}, x_{2}, x_{1}^{2}, x_{1} x_{2}, x_{2}^{2}, x_{1}^{3}, x_{1} x_{2}^{2}, x_{2}^{3}, x_{1}^{4}, x_{1} x_{2}^{3}, x_{2}^{4}\right\}+I .
$$

The combinatorial moment matrix $M_{\mathcal{B}_{2}}(\mathbf{y})$ for $\mathbf{y}=\left(y_{0}, y_{1}, \ldots, y_{11}\right) \in \mathbb{R}^{\mathcal{B}_{4}}$ is

\begin{tabular}{c||c|c|c|c|c|c|} 
& 1 & $x_{1}$ & $x_{2}$ & $x_{1}^{2}$ & $x_{1} x_{2}$ & $x_{2}^{2}$ \\
\hline \hline 1 & $y_{0}$ & $y_{1}$ & $y_{2}$ & $y_{3}$ & $y_{4}$ & $y_{5}$ \\
\hline$x_{1}$ & $y_{1}$ & $y_{3}$ & $y_{4}$ & $y_{6}$ & 1 & $y_{7}$ \\
\hline$x_{2}$ & $y_{2}$ & $y_{4}$ & $y_{5}$ & 1 & $y_{7}$ & $y_{8}$ \\
\hline$x_{1}^{2}$ & $y_{3}$ & $y_{6}$ & 1 & $y_{9}$ & $y_{1}$ & $y_{2}$ \\
\hline$x_{1} x_{2}$ & $y_{4}$ & 1 & $y_{7}$ & $y_{1}$ & $y_{2}$ & $y_{10}$ \\
\hline$x_{2}^{2}$ & $y_{5}$ & $y_{7}$ & $y_{8}$ & $y_{2}$ & $y_{10}$ & $y_{11}$ \\
\hline
\end{tabular}

Theorem 2.5 [9] Assume $\mathcal{B}$ satisfies (4) and $\mathcal{B}_{1}=\left\{1+I, x_{1}+I, \ldots, x_{n}+I\right\}$, and let the coordinates of $\mathbf{y} \in \mathbb{R}^{\mathcal{B}_{2 k}}$ indexed by $\mathcal{B}_{1}$ be $y_{0}, y_{1}, \ldots, y_{n}$. Then $\mathrm{TH}_{k}(I)$ is equal to the closure of the set

$$
\left\{\left(y_{1}, \ldots, y_{n}\right) \mid \mathbf{y} \in \mathbb{R}^{\mathcal{B}_{2 k}} \text { with } M_{\mathcal{B}_{k}}(\mathbf{y}) \succeq 0 \text { and } y_{0}=1\right\} .
$$

When $I=\mathcal{I}(S)$ where $S \subseteq\{0,1\}^{n}$, the closure is not needed and $\mathrm{TH}_{k}(I)$ equals the set (5).

Theorem 2.5 implies that optimizing a linear objective function over $\mathrm{TH}_{k}(I)$ can be reformulated as a semidefinite program with the constraints $M_{\mathcal{B}_{k}}(\mathbf{y}) \succeq 0$ and $y_{0}=1$ which, for fixed $k$, can thus be solved in polynomial time (to any precision).

\subsection{Graphs, cuts and cycles}

Let $G=(V, E)$ be a graph. Throughout, the vertex set is $V=[n]$, the edge set of the complete graph $K_{n}$ is denoted by $E_{n}$, so that $E$ is a subset of $E_{n}$, and the edges of $E_{n}$ correspond to pairs $\{i, j\}$ of distinct vertices $i, j \in V$. For $F \subseteq E, \operatorname{deg}_{F}(v)$ denotes the number of edges of $F$ incident to $v \in V$. A circuit is a set of edges $\left\{\left\{i_{1}, i_{2}\right\},\left\{i_{2}, i_{3}\right\}, \ldots,\left\{i_{t-1}, i_{t}\right\},\left\{i_{t}, i_{1}\right\}\right\}$ where $i_{1}, \ldots, i_{t} \in V$ are pairwise distinct vertices. A set $C \subseteq E$ is a cycle (or Eulerian subgraph) if $\operatorname{deg}_{C}(v)$ is even for all $v \in V$; every non-empty cycle is an edge-disjoint union of circuits. For $S \subseteq V$, the cut $D$ corresponding to the partition $(S, V \backslash S)$ of $V$ is the set of edges $\{i, j\} \in E$ with $|\{i, j\} \cap S|=1$. A basic property is that each cut intersects each cycle in an even number of edges; this is in fact a property of binary matroids which is why we will 
present some of our results later in the more general setting of binary matroids (cf. Sect. 3).

Each cut $D$ can be encoded by its \pm 1 -incidence vector $\chi^{D} \in\{ \pm 1\}^{E}$, called the cut vector of $D$. The cut ideal of $G$, denoted as $I G$, is the vanishing ideal of the set of cut vectors of $G$. The cut polytope of $G$ is

$$
\operatorname{CUT}(G):=\operatorname{conv}\left\{\chi^{D} \mid D \text { is a cut in } G\right\}=\pi_{E}\left(\operatorname{CUT}\left(K_{n}\right)\right) \subseteq \mathbb{R}^{E},
$$

where $\pi_{E}$ is the projection from $\mathbb{R}^{E_{n}}$ onto $\mathbb{R}^{E}$. (Cf. e.g. [5] for an overview on the cut polytope.) The cuts of $K_{n}$ can also be encoded by the cut matrices $X:=\mathbf{x x}^{T}$ for $\mathbf{x} \in\{ \pm 1\}^{n}$ indexing the partitions of $[n]$ corresponding to the cuts. Thus the set

$$
\left\{\mathbf{y} \in \mathbb{R}^{E} \mid \exists X \in \mathbb{R}^{V \times V}, X \succeq 0, X_{i i}=1(i \in V), X_{i j}=y_{\{i, j\}}(\{i, j\} \in E)\right\}
$$

is a relaxation of the cut polytope $\operatorname{CUT}(G)$, over which one can optimize any linear objective function in polynomial time (to any precision), using semidefinite optimization.

Given edge weights $\mathbf{w} \in \mathbb{R}^{E}$, the max-cut problem asks for a cut $D$ in $G$ of maximum total weight $\sum_{e \in D} w_{e}$; thus it can be formulated as

$$
\max \left\{\frac{1}{2} \sum_{e \in E} w_{e}\left(1-y_{e}\right) \mid \mathbf{y} \in \operatorname{CUT}(G)\right\},
$$

where the variable can alternatively be assumed to lie in $\operatorname{CUT}\left(K_{n}\right)$. This is a wellknown NP-hard problem [7]. Thus one is interested in finding tight efficient relaxations of the cut polytope, potentially leading to good approximations for the max-cut problem. It turns out that the simple semidefinite programming relaxation (7) has led to the celebrated 0.878-approximation algorithm of Goemans and Williamson [8] which, as of today, still gives the best known performance guarantee for max-cut.

\section{Theta bodies for cuts and matroids}

In this section we study in detail the hierarchy of SDP relaxations for the cut polytope arising from the theta bodies of the cut ideal. As is well-known, cuts in graphs form a special class of binary matroids. It is thus natural to consider the theta bodies in the more general setting of binary matroids, where the results become more transparent. Then we will apply the results to cuts in graphs (the case of cographic matroids) and also to cycles in graphs (the case of graphic matroids).

\subsection{The cycle ideal of a binary matroid and its theta bodies}

Let $\mathcal{M}=(E, \mathcal{C})$ be a binary matroid; that is, $E$ is a finite set and $\mathcal{C}$ is a collection of subsets of $E$ that is closed under taking symmetric differences. Members of $\mathcal{C}$ are 
called the cycles of $\mathcal{M}$, and members of the set

$$
\mathcal{C}^{*}:=\{D \subseteq E:|D \cap C| \text { even } \forall C \in \mathcal{C}\}
$$

are called the cocycles of $\mathcal{M}$. Then, $\mathcal{M}^{*}=\left(E, \mathcal{C}^{*}\right)$ is again a binary matroid, known as the dual matroid of $\mathcal{M}$, and $\left(\mathcal{M}^{*}\right)^{*}=\mathcal{M}$. The (inclusion-wise) minimal non-empty cycles (cocycles) of $\mathcal{M}$ are called the circuits (cocircuits) of $\mathcal{M}$. An element $e \in E$ is a loop (coloop) of $\mathcal{M}$ if $\{e\}$ is a circuit (cocircuit) of $\mathcal{M}$. Two distinct elements $e, f \in E$ are parallel (coparallel) if $\{e, f\}$ is a circuit (cocircuit) of $\mathcal{M}$. Every non-empty cycle is a disjoint union of circuits. Given $C \in \mathcal{C}$, an element $e \in E \backslash C$ is called a chord of $C$ if there exist $C_{1}, C_{2} \in \mathcal{C}$ such that $C_{1} \cap C_{2}=\{e\}$ and $C=C_{1} \Delta C_{2}$ (if $C$ is a circuit then $C_{1}, C_{2}$ are in fact circuits); $C$ is said to be chordless if it has no chord. Here is a property of chords that we will use later.

Lemma 3.1 Let $C$ be a circuit of $\mathcal{M}$, let $e \in E \backslash C$ be a chord of $C$ and $C_{1}, C_{2}$ be circuits with $C=C_{1} \Delta C_{2}$ and $C_{1} \cap C_{2}=\{e\}$. Then each $C_{i}$ has strictly fewer chords than $C$.

Proof It suffices to show that each chord $e^{\prime}$ of $C_{1}$ is also a chord of $C$. For this let $C_{1}^{\prime}, C_{1}^{\prime \prime}$ be two circuits with $C_{1}^{\prime} \cap C_{1}^{\prime \prime}=\left\{e^{\prime}\right\}$ and $C_{1}=C_{1}^{\prime} \Delta C_{1}^{\prime \prime}$. Say, $e \in C_{1}^{\prime}$, and thus $e \notin C_{1}^{\prime \prime}$. Suppose first that $e^{\prime} \in C_{2}$. Then we have $C_{1}^{\prime \prime} \cap C_{2}=\left\{e^{\prime}\right\}$ and $C_{1}^{\prime \prime} \Delta C_{2} \subseteq C$. As $C$ is a circuit and $C_{1}^{\prime \prime} \neq C_{2}$, we deduce that $C=C_{1}^{\prime \prime} \Delta C_{2}$, which shows that $e^{\prime}$ is a chord of $C$.

Suppose now that $e^{\prime} \notin C_{2}$. Then, $C=C_{1} \Delta C_{2}=\left(C_{1}^{\prime} \Delta C_{2}\right) \Delta C_{1}^{\prime \prime}$ with $\left(C_{1}^{\prime} \Delta C_{2}\right) \cap$ $C_{1}^{\prime \prime}=\left\{e^{\prime}\right\}$, which shows again that $e^{\prime}$ is a chord of $C$.

The binary matroids on $E$ correspond to the $\mathrm{GF}(2)$-vector subspaces of $\mathrm{GF}(2)^{E}$, where $\mathrm{GF}(2)$ is the two-element field $\{0,1\}$ with addition modulo 2 . Namely, identifying a set $F \subseteq E$ with its $0 / 1$-incidence vector $\mathbf{1}^{F} \in \mathrm{GF}(2)^{E}$, the set of cycles $\mathcal{C}$ is a vector subspace of $\operatorname{GF}(2)^{E}$ and the set of cocycles $\mathcal{C}^{*}$ is its orthogonal complement. Thus the cycles of a binary matroid also arise as the solutions in GF(2) ${ }^{E}$ of a linear system $M \mathbf{x}=0$, where $M$ is a matrix with columns indexed by $E$, called a representation matrix of the matroid. In what follows we will use $\mathcal{C}$ (and $\mathcal{C}^{*}$ ) both as a collection of subsets of $E$ and as a $G F(2)$-vector space.

As before let $\mathbb{R} E:=\mathbb{R}\left[x_{e} \mid e \in E\right]$ and, for $C \in \mathcal{C}$, let $\chi^{C} \in\{ \pm 1\}^{E}$ denote its \pm 1 -incidence vector, called its cycle vector. Then,

$$
\operatorname{CYC}(\mathcal{M}):=\operatorname{conv}\left(\chi^{C} \mid C \in \mathcal{C}\right)
$$

is the cycle polytope of $\mathcal{M}$ and

$$
I \mathcal{M}:=\mathcal{I}\left(\chi^{C} \mid C \in \mathcal{C}\right)
$$

is the vanishing ideal of the cycle vectors of $\mathcal{M}$, called the cycle ideal of $\mathcal{M}$. Thus $I \mathcal{M}$ is a real radical zero-dimensional ideal in $\mathbb{R} E$.

We first study the quotient space $\mathbb{R} E / I \mathcal{M}$. For this consider the set

$$
\mathcal{H}:=\left\{x_{e}^{2}-1(e \in E), 1-\mathbf{x}^{D}(D \text { chordless cocircuit of } \mathcal{M})\right\} .
$$


Obviously, $\mathcal{H} \subseteq I \mathcal{M}$; Theorem 3.4 below shows that $\mathcal{H}$ in fact generates the ideal $I \mathcal{M}$. First we observe that $\mathcal{H}$ also generates all binomials $\mathbf{x}^{A}-\mathbf{x}^{B}$ where $A \cup B$ partitions any cocycle of $\mathcal{M}$.

Lemma 3.2 Let $D \in \mathcal{C}^{*}$ be partitioned as $D=A \cup B$. Then, $\mathbf{x}^{A}-\mathbf{x}^{B} \in(\mathcal{H})$.

Proof First we note that it suffices to show that $1-\mathbf{x}^{D} \in(\mathcal{H})$ for all $D \in \mathcal{C}^{*}$. Indeed, for any partition $A \cup B=D, \mathbf{x}^{A}\left(1-\mathbf{x}^{D}\right)=\mathbf{x}^{A}-\left(\mathbf{x}^{A}\right)^{2} \mathbf{x}^{B} \equiv \mathbf{x}^{A}-\mathbf{x}^{B}$ modulo $(\mathcal{H})$. Thus $1-\mathbf{x}^{D} \in(\mathcal{H})$ implies $\mathbf{x}^{A}-\mathbf{x}^{B} \in(\mathcal{H})$.

Next, we show the lemma for the case when $D$ is a cocircuit, using induction on the number $p$ of its chords. If $p=0$ then $1-\mathbf{x}^{D} \in \mathcal{H}$ by definition. So let $p \geq 1$, let $e$ be a chord of $D$ and let $D_{1}, D_{2}$ be cocircuits with $D=D_{1} \Delta D_{2}$ and $D_{1} \cap D_{2}=\{e\}$. Then, $1-\mathbf{x}^{D_{1}}, 1-\mathbf{x}^{D_{2}} \in(\mathcal{H})$, using the induction assumption, since each $D_{i}$ has at most $p-1$ chords by Lemma 3.1. We have: $1-\mathbf{x}^{D} \equiv 1-\left(x_{e}\right)^{2} \mathbf{x}^{D_{1} \backslash\{e\}} \mathbf{x}^{D_{2} \backslash\{e\}}=$ $1-\mathbf{x}^{D_{1}} \mathbf{x}^{D_{2}}=\mathbf{x}^{D_{1}}\left(1-\mathbf{x}^{D_{2}}\right)+1-\mathbf{x}^{D_{1}}$, where the first equality is modulo $(\mathcal{H})$. This shows that $1-\mathbf{x}^{D} \in(\mathcal{H})$.

Finally we show the lemma for $D \in \mathcal{C}^{*}$, using induction on the number $p$ of cocircuits in a partition of $D$. For this, let $D=D_{1} \cup D_{2}$, where $D_{1}$ is a cocircuit and $D_{2}$ is a cocycle partitioned into $p-1$ cocircuits. Then, by the previous case, $1-\mathbf{x}^{D_{1}} \in(\mathcal{H})$, and $1-\mathbf{x}^{D_{2}} \in(\mathcal{H})$ by the induction assumption. Then, $1-\mathbf{x}^{D} \equiv\left(\mathbf{x}^{D_{2}}\right)^{2}-\mathbf{x}^{D_{1}} \mathbf{x}^{D_{2}}=$ $\mathbf{x}^{D_{2}}\left(1-\mathbf{x}^{D_{1}}\right)-\mathbf{x}^{D_{2}}\left(1-\mathbf{x}^{D_{2}}\right)$, where the first equality is modulo $(\mathcal{H})$. This implies $1-\mathbf{x}^{D} \in(\mathcal{H})$.

Define the relation ' $\sim$ ' on $\mathcal{P}(E)$, the collection of all subsets of $E$, by

$$
F \sim F^{\prime} \text { if } F \Delta F^{\prime} \in \mathcal{C}^{*}
$$

this is an equivalence relation, since $\mathcal{C}^{*}$ is closed under taking symmetric differences. The next lemma characterizes the equivalence classes.

Lemma 3.3 For $F, F^{\prime} \subseteq E$, we have:

$$
F \Delta F^{\prime} \in \mathcal{C}^{*} \Longleftrightarrow \mathbf{x}^{F}-\mathbf{x}^{F^{\prime}} \in(\mathcal{H}) \Longleftrightarrow \mathbf{x}^{F}-\mathbf{x}^{F^{\prime}} \in I \mathcal{M} .
$$

Proof If $F \Delta F^{\prime} \in \mathcal{C}^{*}$, then $\mathbf{x}^{F}-\mathbf{x}^{F^{\prime}}=\mathbf{x}^{F \cap F^{\prime}}\left(\mathbf{x}^{F \backslash F^{\prime}}-\mathbf{x}^{F^{\prime} \backslash F}\right) \in(\mathcal{H})$, using Lemma 3.2; $\mathbf{x}^{F}-\mathbf{x}^{F^{\prime}} \in(\mathcal{H}) \Longrightarrow \mathbf{x}^{F}-\mathbf{x}^{F^{\prime}} \in I \mathcal{M}$ follows from $\mathcal{H} \subseteq I \mathcal{M}$. Conversely, if $\mathbf{x}^{F}-\mathbf{x}^{F^{\prime}} \in I \mathcal{M}$ then, for any $C \in \mathcal{C}, \mathbf{x}^{F}-\mathbf{x}^{F^{\prime}}$ vanishes at $\chi^{C}$ and thus $|C \cap F|$ and $\left|C \cap F^{\prime}\right|$ have the same parity, which implies that $\left|C \cap\left(F \Delta F^{\prime}\right)\right|$ is even and thus $F \Delta F^{\prime} \in \mathcal{C}^{*}$.

Let

$$
\mathcal{F}:=\left\{F_{1}, \ldots, F_{N}\right\}
$$

be a set of distinct representatives of the equivalence classes of $\mathcal{P}(E) / \sim$ and set

$$
\mathcal{B}:=\left\{\mathbf{x}^{F}+I \mathcal{M} \mid F \in \mathcal{F}\right\}
$$


Theorem 3.4 The set $\mathcal{B}$ is a basis of the vector space $\mathbb{R} E / I \mathcal{M}$ and the set $\mathcal{H}$ generates the ideal IM.

Proof First, we show that $\mathcal{B}$ spans the space $\mathbb{R} E /(\mathcal{H})$. As $x_{e}^{2}-1 \in \mathcal{H}(\forall e \in E)$, it suffices to show that $\mathcal{B}$ spans all cosets of square-free monomials. For this, let $F \subseteq E$ and, say, $F \sim F_{1}$; then, $\mathbf{x}^{F}-\mathbf{x}^{F_{1}} \in I \mathcal{M}$ by Lemma 3.3 , which shows that $\mathbf{x}^{F}+I \mathcal{M} \in \operatorname{span}(\mathcal{B})$. Therefore, we obtain:

$$
|\mathcal{C}|=\operatorname{dim} \mathbb{R} E / I \mathcal{M} \leq \operatorname{dim} \mathbb{R} E /(\mathcal{H}) \leq|\mathcal{B}|=N .
$$

To conclude the proof it now suffices to show that $|\mathcal{C}|=N$. For this, fix a basis $\left\{C_{1}, \ldots, C_{m}\right\}$ of the $\mathrm{GF}(2)$-vector space $\mathcal{C}$, so that $|\mathcal{C}|=2^{m}$. Let $M$ be the $m \times|E|$ matrix whose rows are the $0 / 1$-incidence vectors of $C_{1}, \ldots, C_{m}$. Then $M \mathbf{x}$ takes $2^{m}$ distinct values for all $\mathbf{x} \in \mathrm{GF}(2)^{E}$. As, for $F, F^{\prime} \subseteq E, F \sim F^{\prime}$ if and only if $M 1^{F}=M 1^{F^{\prime}}$, we deduce that the equivalence relation (10) has $N=2^{m}$ equivalence classes.

We now consider the combinatorial moment matrices for the cycle ideal $I \mathcal{M}$. For any integer $k$ define the set

$$
\mathcal{F}_{k}:=\left\{F \in \mathcal{F} \mid \exists D \in \mathcal{C}^{*} \text { with }|F \Delta D| \leq k\right\}
$$

corresponding to the equivalence classes of $\sim$ having a representative of cardinality at most $k$. Then $\mathcal{B}_{k}=\left\{\mathbf{x}^{F}+I \mathcal{M} \mid F \in \mathcal{F}_{k}\right\}$ can be identified with the set $\mathcal{F}_{k}$. Moreover relation (4) holds, so that the entries of the truncated moment matrix $M_{\mathcal{B}_{k}}(\mathbf{y})$ depend only on the entries of $\mathbf{y}$ indexed by $\mathcal{B}_{2 k}$. For instance, $\mathcal{F}_{1}$ can be any maximal subset of $E$ containing no coloops or coparallel elements of $\mathcal{M}$, along with $\emptyset$. Indeed, $e \in E$ is a coloop precisely if $\{e\} \sim \emptyset$, and two elements $e \neq f \in E$ are coparallel precisely if $e \sim f$. Thus, $\mathcal{F}_{0}=\{\emptyset\}$ and $\mathcal{F}_{1} \backslash \mathcal{F}_{0}=E$ if $\mathcal{M}$ has no coloops and no coparallel elements.

When $\mathcal{M}$ has no coloops and no coparallel elements, its $k$-th theta body $\mathrm{TH}_{k}(I \mathcal{M})$ consists of the vectors $\mathbf{y} \in \mathbb{R}^{E}$ for which there exists a positive semidefinite $\left|\mathcal{F}_{k}\right| \times\left|\mathcal{F}_{k}\right|$ matrix $X$ satisfying $X_{\emptyset, e}=y_{e}$ for all $e \in E$ and

(i) $X_{\emptyset, \emptyset}=1$,

(ii) $X_{F_{1}, F_{2}}=X_{F_{3}, F_{4}}$ if $F_{1} \Delta F_{2} \Delta F_{3} \Delta F_{4} \in \mathcal{C}^{*}$.

Remark 3.5 The constraints (14)(ii) contain in particular the constraints

$$
X_{F_{1}, F_{2}}=X_{F_{3}, F_{4}} \text { if } F_{1} \Delta F_{2}=F_{3} \Delta F_{4} .
$$

Note that the above constraints are the basic 'moment constraints', which are satisfied by all \pm 1 vectors. Indeed, if $\mathbf{y}=\chi^{F} \in\{-1,1\}^{E}$, define the $\left|\mathcal{F}_{k}\right| \times\left|\mathcal{F}_{k}\right|$ matrix $X$ by $X_{F_{1}, F_{2}}:=(-1)^{\left|F \cap F_{1}\right|}(-1)^{\left.\mid F \cap F_{2}\right) \mid}$, so that $y_{e}=X_{\emptyset, e}(e \in E)$. Then $X \succeq 0$ since $X=\mathbf{u u}^{T}$ where $\mathbf{u}=\left((-1)^{\left|F \cap F_{i}\right|}\right)_{F_{i} \in \mathcal{F}_{k}}$, and $X$ satisfies (14) (i) and (15). Therefore the constraints (15) do not cut off any point of the cube $[-1,1]^{E}$. Non-trivial constraints that cut off points of $[-1,1]^{E}$ that do not lie in $\operatorname{CYC}(\mathcal{M})$ come from those constraints (14)(ii) where $F_{1} \Delta F_{2} \Delta F_{3} \Delta F_{4}$ is a non-empty cocycle. 


\subsection{Application to cuts in graphs}

Binary matroids arise naturally from graphs in the following way. Let $G=([n], E)$ be a graph, let $\mathcal{C}_{G}$ denote its collection of cycles, and $\mathcal{D}_{G}$ its collection of cuts. Since $\mathcal{C}_{G}$ and $\mathcal{D}_{G}$ are closed under symmetric difference, both $\mathcal{M}_{G}:=\left(E, \mathcal{C}_{G}\right)$ and $\mathcal{M}_{G}^{*}:=$ $\left(E, \mathcal{D}_{G}\right)$ are binary matroids, and since each cut has an even intersection with each cycle, they are duals of each other. The matroid $M_{G}$ is known as the graphic matroid of $G$ and $\mathcal{M}_{G}^{*}$ as its cographic matroid.

We consider here the case when $\mathcal{M}=\mathcal{M}_{G}^{*}$ is the cographic matroid of $G=$ $([n], E)$. Then, $\operatorname{CYC}(\mathcal{M})=\operatorname{CUT}(G)$ is the cut polytope of $G$ and $I \mathcal{M}$ is the cut ideal of $G$ (denoted earlier by $I G$ ), thus defined as the vanishing ideal of all cut vectors in $G$.

So $I G$ is an ideal in $\mathbb{R} E$, while $I K_{n}$ is an ideal in $\mathbb{R} E_{n}$. One can easily verify that $I G$ is the elimination ideal, $I K_{n} \cap \mathbb{R} E$, of $I K_{n}$ with respect to $E$. By Theorem 3.4, we know that the (edge) binomials $x_{e}^{2}-1(e \in E)$ together with the binomials $1-\mathbf{x}^{C}$ ( $C$ chordless circuit of $G$ ) generate the cut ideal $I G$. When $G=K_{n}$ is a complete graph, the only chordless circuits are the triangles so that, beside the edge binomials, it suffices to consider the binomials $1-x_{\{i, j\}} x_{\{i, k\}} x_{\{j, k\}}\left(\right.$ or $x_{\{i, j\}}-x_{\{i, k\}} x_{\{j, k\}}$ ) for distinct $i, j, k \in[n]$.

When $G$ is connected, there are $2^{n-1}$ distinct cuts in $G$ (corresponding to the partitions of $[n]$ into two classes) and, when $G$ has $p$ connected components, there are $2^{n-p}$ cuts in $G$ and thus $\operatorname{dim} \mathbb{R} E / I G=2^{n-p}$.

The following notion of $T$-joins arises naturally when considering the equivalence relation (10). Given a set $T \subseteq[n]$, a set $F \subseteq E$ is called a $T$-join if $T=\{v \in[n] \mid$ $\operatorname{deg}_{F}(v)$ is odd $\}$. For instance, the $\emptyset$-joins are the cycles of $G$ and, for $T=\{s, t\}$, the minimum $T$-joins correspond to the shortest $s-t$ paths in $G$. If $F$ is a $T$-join and $F^{\prime}$ is $T^{\prime}$-join, then $F \Delta F^{\prime}$ is a $\left(T \Delta T^{\prime}\right)$-join. In particular, $F \sim F^{\prime}$, i.e. $F \Delta F^{\prime} \in \mathcal{C}_{G}$, precisely when $F, F^{\prime}$ are both $T$-joins for the same $T \subseteq[n]$.

Thus the equivalence classes of $\sim$ correspond to the members of the set $\mathcal{T}_{G}:=\{T \subseteq$ [n] $\mid \exists T$-join in $G\}$ (which consists of the sets $T_{1} \cup \ldots \cup T_{p}$, where each $T_{i}$ is an even subset of $V_{i}$ and $V_{1}, \ldots, V_{p}$ are the connected components of $G$ ). The set $\mathcal{F}$ (in (11)) consists of one $T$-join $F_{T}$ for each $T \in \mathcal{T}_{G}$, and $\mathcal{F}_{k}=\left\{F_{T} \mid T \in \mathcal{T}_{k}\right\}$, after defining $\mathcal{T}_{k}$ as the set of all $T \in \mathcal{T}_{G}$ for which there exists a $T$-join of size at most $k$. Then the corresponding basis of $\mathbb{R} E / I G$ is $\mathcal{B}=\left\{\mathbf{x}^{F_{T}}+I G \mid T \in \mathcal{T}_{G}\right\}, \mathcal{B}_{k}=\left\{\mathbf{x}^{F_{T}}+I G \mid T \in \mathcal{T}_{k}\right\}$ and (4) holds.

For instance, $\mathcal{F}_{1}$ consists of all edges $e \in E$ together with the empty set. Hence the first order theta body $\mathrm{TH}_{1}(I G)$ consists of the vectors $\mathbf{y} \in \mathbb{R}^{E}$ for which there exists a positive semidefinite matrix $X$ indexed by $E \cup\{\emptyset\}$ satisfying $y_{e}=X_{\emptyset, e}(e \in E)$ and

(i) $X_{\emptyset, \emptyset}=X_{e, e}=1$ for all $e \in E$,

(ii) $X_{e, f}=X_{\emptyset, g}$ if $\{e, f, g\}$ is a triangle in $G$,

(iii) $X_{e, f}=X_{g, h}$ if $\{e, f, g, h\}$ is a circuit in $G$.

Remark 3.6 When $G=K_{n}$ is the complete graph, for any even $T \subseteq[n]$, the minimum cardinality of a $T$-join is $|T| / 2$; just choose for $F_{T}$ a set of $|T| / 2$ disjoint edges 
(i.e. a perfect matching) on $T$. Hence the set $\mathcal{T}_{k}$ consists of all even $T \subseteq[n]$ with $|T| \leq 2 k$. As an illustration, if we index the combinatorial moment matrices by $\mathcal{T}_{k}$, then the condition (14) (ii) reads:

$$
X_{T_{1}, T_{2}}=X_{T_{3}, T_{4}} \text { if } T_{1} \Delta T_{2}=T_{3} \Delta T_{4}
$$

This observation will enable us to relate the theta body hierarchy to the semidefinite relaxations of the cut polytope considered in [15], cf. Sect. 3.3.

Example 3.7 If $G$ has no circuit of length 3 or 4 , then $\mathrm{TH}_{1}(I G)=[-1,1]^{E}$, since the conditions (16)(ii)-(iii) are void. For instance, if $G$ is a forest, then $\mathrm{TH}_{1}(I G)=$ $[-1,1]^{E}=\operatorname{CUT}(G)$ and thus $I G$ is $\mathrm{TH}_{1}$-exact. On the other hand, if $G=C_{n}$ is a circuit of length $n \geq 5$, then $\mathrm{TH}_{1}(I G)=[-1,1]^{E}$ strictly contains the polytope $\operatorname{CUT}\left(C_{n}\right)$ (as $|E|=n$ and $\operatorname{CUT}\left(C_{n}\right)$ has only $2^{n-1}$ vertices). Thus $I C_{n}$ is not $\mathrm{TH}_{1-}$ exact for $n \geq 5$.

Example 3.8 For $G=K_{5}, I K_{5}$ is not $\mathrm{TH}_{1}$-exact. Indeed, the inequality $\sum_{e \in E_{5}} x_{e}+$ $2 \geq 0$ induces a facet of $\operatorname{CUT}\left(K_{5}\right)$ (cf. e.g. [5, Chap. 28.2]) and the linear form $\sum_{e \in E_{5}} x_{e}+2$ takes three distinct values on the vertices of $\operatorname{CUT}\left(K_{5}\right)$ (namely, 0 on the facet, 12 on the trivial empty cut and 4 on the cut obtained by separating a vertex from all the others). Applying Theorem 2.2, we can conclude that $I K_{5}$ is not $\mathrm{TH}_{1}$-exact.

In Sect. 3.3 below we will characterize the graphs whose cut ideals are $\mathrm{TH}_{1}$-exact and we will determine the precise order $k$ at which the cut ideal of a circuit is $\mathrm{TH}_{k}$-exact in Sect. 5.

\subsection{Comparison with other SDP relaxations of the cut polytope}

We mention here the link between the theta bodies of the cut ideal $I G$ and some other semidefinite relaxations of the cut polytope $\operatorname{CUT}(G)$. First note that the relaxation $\mathrm{TH}_{1}(I G)$ coincides with the edge-relaxation considered by Rendl and Wiegele (see [25]) and numerical experiments there indicates that it is often tighter than the basic semidefinite relaxation (7) of $\operatorname{CUT}(G)$.

Next we compare the theta bodies of $I G$ with the relaxations $Q_{t}(G)$ of $\operatorname{CUT}(G)$ considered in [15]. ${ }^{1}$ For $t \in \mathbb{N}$, set $\mathcal{O}_{t}(n):=\{T \subseteq[n]|| T \mid \leq t$ and $|T| \equiv t$ $\bmod 2\}$. Then $Q_{t}(G)$ consists of the vectors $\mathbf{y} \in \mathbb{R}^{E}$ for which there exists a positive semidefinite matrix $X$ indexed by $\mathcal{O}_{t}(n)$ satisfying (17), $X_{T, T}=1\left(T \in \mathcal{O}_{t}(n)\right)$, and $y_{\{i, j\}}=X_{\emptyset,\{i, j\}}$ for $t$ even (resp., $y_{\{i, j\}}=X_{\{i\},\{j\}}$ for $t$ odd) for all edges $\{i, j\} \in E$. Therefore, for $t=1, Q_{1}(G)$ coincides with the Goemans-Williamson SDP relaxation (7). Moreover, for even $t=2 k, Q_{2 k}\left(K_{n}\right)$ coincides with the theta body $\operatorname{TH}_{k}\left(I K_{n}\right)$. (To see it use Remark 3.6.) The following chain of inclusions shows the link to the theta bodies:

$$
\operatorname{CUT}(G) \subseteq Q_{2 k}(G)=\pi_{E}\left(Q_{2 k}\left(K_{n}\right)\right)=\pi_{E}\left(\mathrm{TH}_{k}\left(I K_{n}\right)\right) \subseteq \mathrm{TH}_{k}(I G)
$$

\footnotetext{
${ }^{1}$ For simplicity in the notation we shift the indices by 1 with respect to [15].
} 
(where the last inclusion follows using (2)). Therefore, the $k$-th theta body $\mathrm{TH}_{k}(I G)$ is in general a weaker relaxation than $Q_{2 k}(G)$. For instance, for the 5-circuit, $\operatorname{CUT}\left(C_{5}\right)=$ $Q_{2}\left(C_{5}\right)$ (see [15]) but CUT $\left(C_{5}\right)$ is strictly contained in $\mathrm{TH}_{1}\left(I C_{5}\right)=[-1,1]^{5}$ (see Example 3.7).

On the other hand, the SDP relaxation $\mathrm{TH}_{k}(I G)$ can be much simpler and less costly to compute than $Q_{2 k}(G)$, since its definition exploits the structure of $G$ and thus often uses smaller matrices. Indeed, $Q_{2 k}(G)$ is defined as the projection of $Q_{2 k}\left(K_{n}\right)$, whose definition involves matrices indexed by all even sets $T \subseteq[n]$ of size at most $2 k$, thus not depending on the structure of $G$. On the other hand, the matrices needed to define $\mathrm{TH}_{k}(I G)$ are indexed by the even sets $T \subseteq[n]$ of size at most $2 k$ for which $G$ has a $T$-join of size at most $k$. This can be checked efficiently since the minimum weight $T$-join problem has a polynomial time algorithm (cf. [6]). For instance, for $k=1, \mathrm{TH}_{1}(I G)$ uses matrices of size $1+|E|$, while $Q_{2}(G)$ needs matrices of size $1+\left(\begin{array}{l}n \\ 2\end{array}\right)$. We refer to Sect. 3.5 for more details on the complexity of constructing the above theta bodies.

Example 3.9 It was shown in [14] that CUT $\left(K_{n}\right)$ is strictly contained in $Q_{k}\left(K_{n}\right)$ for $k<\left\lceil\frac{n}{2}\right\rceil-1$. Therefore, $\operatorname{CUT}\left(K_{n}\right) \subset \mathrm{TH}_{k}\left(I K_{n}\right)=Q_{2 k}\left(K_{n}\right)$ for all $2 k<\left\lceil\frac{n}{2}\right\rceil-1$. This implies that $I K_{n}$ is not $\mathrm{TH}_{k}$-exact for $k \leq\left\lfloor\frac{n-1}{4}\right\rfloor$. However, it is known that $\operatorname{CUT}\left(K_{n}\right)=Q_{\left\lceil\frac{n}{2}\right\rceil}\left(K_{n}\right)$ when $n \leq 7$. Therefore, $I K_{5}, I K_{6}$ and $I K_{7}$ are all $\mathrm{TH}_{2-}$ exact.

For some graphs there is a special inclusion relationship between the theta bodies and the $Q_{t}$-hierarchy. We consider first graphs with bounded diameter.

Lemma 3.10 Let $G$ be a graph with diameter at most $k$, i.e., such that any two vertices can be joined by a path traversing at most $k$ edges. Then $\mathrm{TH}_{k}(I G) \subseteq Q_{2}(G)$.

Proof It suffices to observe that the set $\mathcal{T}_{k}$ indexing the matrices in the definition of $\mathrm{TH}_{k}(I G)$ (which consists of the even sets $T \subseteq V$ for which there is a $T$-join of size at most $k$ ) contains all pairs of vertices. Thus $\mathcal{T}_{k}$ contains the set $\mathcal{O}_{2}(n)$ indexing the matrices in the definition of $Q_{2}(G)$.

Next we observe that $\mathrm{TH}_{k}(I G)$ refines the Goemans-Williamson relaxation (7) for graphs with radius $k$.

Lemma 3.11 Let $G$ be a graph with radius at most $k$, i.e., there exists a vertex that can be joined to any other vertex by a path traversing at most $k$ edges. Then $\mathrm{TH}_{k}(I G) \subseteq$ $Q_{1}(G)$.

Proof Say vertex 1 can be joined to all other vertices $i \in[n] \backslash\{1\}$ by a path of length at most $k$. Then the set $\mathcal{T}_{k}$ contains $\emptyset,\{i, j\}$ for all edges $i j \in E$, and all pairs $\{1, i\}$ for $i \in[n] \backslash\{1\}$. Let $\mathbf{y} \in \mathrm{TH}_{k}(I G)$, i.e. there exists a positive semidefinite matrix $X$ indexed by $\mathcal{T}_{k}$ satisfying (17) and $y_{e}=X_{\emptyset, e}$ for $e \in E$. Consider the $n \times n$ matrix $Y$ defined by $Y_{i i}=1(i \in[n]), Y_{1 i}=X_{\emptyset,\{1, i\}}(i \in[n] \backslash\{1\})$, and $Y_{i j}=X_{\{1, i\},\{1, j\}}$ $(i \neq j \in[n] \backslash\{1\}$ ). Then $Y \succeq 0$ (since $Y$ coincides with the principal submatrix of $X$ indexed by $\emptyset,\{1,2\}, \ldots,\{1, n\}), y_{\{i, j\}}=Y_{i j}$ for all $\{i, j\} \in E$ (using (17)). This shows $\mathbf{y} \in Q_{1}(G)$, concluding the proof. 
In particular, as already noted in [25], $\mathrm{TH}_{1}(I G) \subseteq Q_{1}(G)$ if $G$ contains a vertex adjacent to all other vertices. For an arbitrary graph $G$, let $G^{*}$ be the graph obtained by adding edges to $G$ so that one of its vertices is adjacent to all other vertices. Thus, $\mathrm{TH}_{1}\left(I G^{*}\right) \subseteq Q_{1}\left(G^{*}\right)$ by Lemma 3.11. Taking projections onto the edge set of $G$, the relaxation $\pi_{E}\left(\mathrm{TH}_{1}\left(I G^{*}\right)\right)$ is contained in $\pi_{E}\left(Q_{1}\left(G^{*}\right)\right)=Q_{1}(G)$ (and in $\left.\mathrm{TH}_{1}(I G)\right)$.

\subsection{Application to circuits in graphs}

Let us consider briefly the case when $\mathcal{M}=\mathcal{M}_{G}$ is the graphic matroid of a graph $G=(V, E)$, i.e. $\mathcal{C}=\mathcal{C}_{G}$ is the collection of cycles of $G$ and $\mathcal{C}^{*}=\mathcal{D}_{G}$ is its collection of cuts.

One can find a set $\mathcal{F}$ of representatives for the equivalence classes of (10) as follows. Namely, assume for simplicity that $G$ is connected and let $E_{0} \subseteq E$ be the edge set of a spanning tree in $G$. Then the collection $\mathcal{F}:=\mathcal{P}\left(E \backslash E_{0}\right)$ is a set of distinct representatives for the classes of (10). Indeed, note first that no two distinct subsets $F, F^{\prime}$ of $E \backslash E_{0}$ are in relation by $\sim$, since each non-empty cut meets the tree $E_{0}$. Next, any subset $X \subseteq E_{0}$ determines a unique cut $D_{X}$ for which $D_{X} \cap E_{0}=X$, so that $X \sim X \Delta D_{X}$. Hence, for any set $Z \subseteq E$, write $Z=X \cup Y$ with $X \subseteq E_{0}$ and $Y \subseteq E \backslash E_{0}$; then $Z \sim X \Delta D_{X} \Delta Y$ is thus in the same equivalence class as a subset of $E \backslash E_{0}$.

Note however that the above set $\mathcal{F}$ may not consist of the minimum cardinality representatives. In fact, as we observe in the next section, finding a minimum cardinality representative in each equivalence class amounts to solving a maximum weight cut problem, thus a hard problem. Nevertheless this collection $\mathcal{F}$ can be used to index truncated moment matrices (simply index the $k$-th order matrix by all $F \in \mathcal{F}$ with $|F| \leq k)$. However, studying this SDP hierarchy is less relevant for optimization purposes since the linear inequality description of $\mathrm{CYC}\left(\mathcal{M}_{G}\right)$ is completely known (see Theorem 4.4 below), and one can find a maximum weight cycle in a graph in polynomial time (cf. [6]).

\subsection{Computational complexity of theta bodies}

We group here some observations about the computational complexity of building the matrices $M_{\mathcal{B}_{k}}(\mathbf{y})$ needed to define the theta body $\mathrm{TH}_{k}(I \mathcal{M})$ of a binary matroid $\mathcal{M}$.

The computation of the matrix $M_{\mathcal{B}_{k}}(\mathbf{y})$ is done in two steps:

- First compute the sets $\mathcal{F}_{k}$ and $\mathcal{F}_{2 k}$.

- Then compute the entries of $M_{\mathcal{B}_{k}}(\mathbf{y})$, i.e., for all $F, F^{\prime} \in \mathcal{F}_{k}$, find $F^{\prime \prime} \in \mathcal{F}_{2 k}$ such that $F \Delta F^{\prime} \sim F^{\prime \prime}$, i.e., $F \Delta F^{\prime} \Delta F^{\prime \prime} \in \mathcal{C}^{*}$.

As we see below both steps involve making repeated calls to a membership oracle for $\mathcal{C}^{*}$. Such a membership oracle gives a yes/no answer to any query of the form:

Given $X \subseteq E$, does $X$ belong to $\mathcal{C}^{*}$ ? 
If the binary matroid $\mathcal{M}$ is given by a representation matrix $M$, then it is easy to answer such a query. Indeed, first bring $M$ into the form $M=\left(I_{r} A\right)$, where $A \in\{0,1\}^{r \times s}$ (using Gaussian elimination). Then $M^{*}:=\left(A^{T} I_{S}\right)$ is a representing matrix for $\mathcal{M}^{*}$ and thus $X \in \mathcal{C}^{*}$ if and only if $M^{*} \mathbf{1}^{X}=0$ modulo 2 .

In the case when $\mathcal{M}$ is the cographic matroid of a graph $G=(V, E)$, checking membership in $\mathcal{C}^{*}$ is easy: $X \in \mathcal{C}^{*}$ (i.e., $X$ is a cycle of $G$ ) if and only if each node $v \in V$ is adjacent to an even number of edges of $X$.

We first indicate how to construct the set $\mathcal{F}_{k}$. Say $\mathcal{M}=(E, \mathcal{C})$ is a binary matroid on $E, m:=|E|$, and $\mathcal{P}_{k}(E)$ denotes the collection of all subsets of $E$ with cardinality at most $k$. As in (11), $\mathcal{F}$ denotes a set of distinct representatives for the equivalence classes of the relation $\sim$ in (10), and $\mathcal{F}_{k}$ is (as in (13)) a set of distinct representatives for those classes that contain at least one set of cardinality at most $k$. For any $F \subseteq E$, check that the subsets of $E$ that are equivalent to $F$ are precisely those of the form $F \Delta D, D \in \mathcal{C}^{*}$, and that for two distinct elements $D_{1}$ and $D_{2}$ in $\mathcal{C}^{*}, F \Delta D_{1} \neq F \Delta D_{2}$. Therefore, $|\mathcal{F}|=2^{m} /\left|\mathcal{C}^{*}\right|$ and

$$
\left|\mathcal{F}_{k}\right| \leq\left|\mathcal{P}_{k}(E)\right|=\sum_{i=0}^{k}\left(\begin{array}{c}
m \\
i
\end{array}\right)=O\left(m^{k}\right)
$$

Such a set $\mathcal{F}_{k}$ can be constructed using the following simple procedure: Order the elements of $E$ as $e_{1}, \ldots, e_{m}$ and order the elements of $\mathcal{P}_{k}(E)$ by increasing cardinalities as $\emptyset,\left\{e_{1}\right\}, \ldots,\left\{e_{m}\right\},\left\{e_{1}, e_{2}\right\}, \ldots,\left\{e_{m-1}, e_{m}\right\}, \ldots,\left\{e_{1}, \ldots, e_{k}\right\}, \ldots,\left\{e_{m-k+1}, \ldots, e_{m}\right\}$, denoted as $X_{1}=\emptyset, X_{2}, X_{3}, \ldots$ We successively scan the elements $X_{1}, X_{2}, \ldots$ and decide which ones should be selected in $\mathcal{F}_{k}$ in the following way: First select $F_{1}:=X_{1}$. Then, say $X_{1}, \ldots, X_{i}$ have been scanned and we have selected $\left\{F_{1}, \ldots, F_{r}\right\} \subseteq$ $\left\{X_{1}, \ldots, X_{i}\right\}$; if $X_{i+1} \Delta F_{s} \notin \mathcal{C}^{*} \forall s=1, \ldots, r$, then $F_{r+1}:=X_{i+1}$ is selected; otherwise we do not select it; go on with next set $X_{i+2}$.

Let $F_{1}, \ldots, F_{p}$ be the sets which have been selected by this procedure. Then, $F_{r} \nsim F_{r^{\prime}}$ for $1 \leq r<r^{\prime} \leq p$, and any $X \in \mathcal{P}_{k}(E)$ is equal or equivalent to one of $F_{1}, \ldots, F_{p}$. Therefore, the set $\left\{F_{1}, \ldots, F_{p}\right\}$ constitutes a set of distinct representatives for the classes of $\sim$ containing some set of size at most $k$ and thus it can be chosen for $\mathcal{F}_{k}$. Moreover it has the property that any member of $\mathcal{F}_{k}$ has the smallest cardinality in its equivalence class.

The above procedure makes a number of calls to a membership oracle in $\mathcal{C}^{*}$ which is of order $O\left(m^{k}\right)$, thus polynomial in $m=|E|$, when $k$ is fixed.

Next we see how to construct the entries of $M_{\mathcal{B}_{k}}(\mathbf{y})$. For this we need to build the multiplication table: for any $F, F^{\prime} \in \mathcal{F}_{k}$, we must find the element $F^{\prime \prime} \in \mathcal{F}$ for which $\mathbf{x}^{F} \mathbf{x}^{F^{\prime}}=\mathbf{x}^{F^{\prime \prime}}$ modulo $I \mathcal{M}$ or, equivalently, $F \Delta F^{\prime} \sim F^{\prime \prime}$. As $\left|F \Delta F^{\prime}\right| \leq 2 k, F^{\prime \prime} \in$ $\mathcal{F}_{2 k}$. Therefore, in order to build $M_{\mathcal{B}_{k}}(\mathbf{y})$ it suffices to build the set $\mathcal{F}_{2 k}$ which, for fixed $k$, can be done with a polynomial number of calls to a membership oracle for $\mathcal{C}^{*}$.

Remark 3.12 On the other hand, let us point out that, given $F \subseteq E$, the problem of finding a representative $F^{\prime}$ of minimum cardinality in the equivalence class of $F$ is hard in general. (Of course, if we fix the cardinality of $F$ the problem becomes easy 
as we just observed.) Indeed this is the problem:

$$
\min |D \Delta F| \text { such that } D \in \mathcal{C}^{*}
$$

or equivalently

$$
\max \mathbf{w}^{T} \mathbf{x} \text { such that } \mathbf{x} \in \operatorname{CYC}\left(\mathcal{M}^{*}\right)
$$

after defining $\mathbf{w} \in \mathbb{R}^{E}$ by $w_{e}=-1$ for $e \in F$ and $w_{e}=1$ for $e \in E \backslash F$ (and noting that $\left.\mathbf{w}^{T} \chi^{D}=|E|-2|F \Delta D|\right)$.

When $\mathcal{M}$ is a cographic matroid, the above problem (19) asks to find a minimum cardinality $T$-join (where $T$ is the set of odd degree nodes in $F$ ) which can be solved in polynomial time (see [6]).

When $\mathcal{M}$ is a graphic matroid, the above problem (20) is an instance of the maximum cut problem, which is an NP-hard problem for general graphs. However (20) is polynomial time solvable if $\mathcal{M}$ has no $\mathcal{M}\left(K_{5}\right)$-minor (see [1]).

The problem (20) is also polynomial time solvable when $\mathcal{M}$ or $\mathcal{M}^{*}$ does not have $F_{7}$ or $\mathcal{M}\left(K_{5}\right)^{*}$ as a minor; see [11] for details and for other classes of matroids for which (20) is polynomial time solvable.

\section{Matroids whose cycle ideals are $\mathbf{T H}_{1}$-exact}

\subsection{Matroid minors}

Let $\mathcal{M}=(E, \mathcal{C})$ be a binary matroid and $e \in E$. Set

$$
\mathcal{C} \backslash e:=\{C \in \mathcal{C} \mid e \notin C\}, \quad \mathcal{C} / e:=\{C \backslash\{e\} \mid C \in \mathcal{C}\}
$$

Then, $\mathcal{M} \backslash e:=(E \backslash\{e\}, \mathcal{C} \backslash e)$ and $\mathcal{M} / e:=(E \backslash\{e\}, \mathcal{C} / e)$ are again binary matroids; one says that $\mathcal{M} \backslash e$ is obtained by deleting $e$ and $\mathcal{M} / e$ by contracting $e$. A minor of $\mathcal{M}$ is obtained by a sequence of deletions and contractions, thus of the form $\mathcal{M} \backslash X / Y$ for disjoint $X, Y \subseteq E$. In the language of binary spaces, $C \backslash e$ arises from $\mathcal{C}$ by taking the intersection with the hyperplane $x_{e}=0$, while $\mathcal{C} / e$ arises by projecting $\mathcal{C}$ onto $\mathbb{R}^{E \backslash\{e\}}$.

Example 4.1 Let $M_{r}$ denote the $r \times\left(2^{r}-1\right)$ matrix whose columns are all nonzero 0/1 vectors of length $r$, and let $\mathcal{P}_{r}$ denote the binary matroid represented by $M_{r}$, called the binary projective space of dimension $r-1$. One can verify that $\mathcal{P}_{r}$ has $2^{r}$ cocycles; the non-empty cocycles have size $2^{r-1}$ and thus are cocircuits. Hence, $\operatorname{CYC}\left(\mathcal{P}_{r}^{*}\right)$ is a simplex and $I \mathcal{P}_{r}^{*}$ is $\mathrm{TH}_{1}$-exact. When $n=3, \mathcal{P}_{3}=: F_{7}$ is called the Fano matroid. It will follow from Theorem 4.6 that $I F_{7}$ is also $\mathrm{TH}_{1}$ exact. 
Example $4.2 R_{10}$ is the binary matroid on 10 elements, represented by the matrix

$$
\begin{aligned}
& \begin{array}{llllllllll}
34 & 35 & 45 & 23 & 24 & 25 & 13 & 14 & 15 & 12
\end{array} \\
& \left(\begin{array}{llllllllll}
1 & 1 & 1 & 1 & 1 & 1 & 0 & 0 & 0 & 0 \\
1 & 1 & 1 & 0 & 0 & 0 & 1 & 1 & 1 & 0 \\
1 & 0 & 0 & 1 & 1 & 0 & 1 & 1 & 0 & 1 \\
0 & 1 & 0 & 1 & 0 & 1 & 1 & 0 & 1 & 1 \\
0 & 0 & 1 & 0 & 1 & 1 & 0 & 1 & 1 & 1
\end{array}\right),
\end{aligned}
$$

where it is convenient to index the columns by the edge set $E_{5}$ of $K_{5}$. Then the cycles of $R_{10}$ correspond to the even cycles of $K_{5}$, and the cocycles of $R_{10}$ to the cuts of $K_{5}$ and their complements. Note that $R_{10}$ is isomorphic to its dual. Consider the inequality:

$$
\sum_{e \in F} x_{e}-\sum_{e \in E_{5} \backslash F} x_{e} \geq-4
$$

where $F$ consists of three edges adjacent to a common vertex (e.g. $F=\{12,13,14\})$. (Thus (21) is of the form (22), but with a shifted right hand side.) One can verify that (21) defines a facet of $\mathrm{CYC}\left(R_{10}\right)$ and that the linear function in (21) takes three distinct values on the cycles of $R_{10}$ (namely, 0,4 , and -4). Therefore, in view of Theorem 2.2, we can conclude that $R_{10}$ is not $\mathrm{TH}_{1}$-exact.

\subsection{The cycle polytope}

As each cycle and cocycle have an even intersection, the following inequalities are valid for the cycle polytope $\mathrm{CYC}(\mathcal{M})$ :

$$
\sum_{e \in F} x_{e}-\sum_{e \in D \backslash F} x_{e} \geq 2-|D| \text { for } D \in \mathcal{C}^{*}, F \subseteq D,|F| \text { odd }
$$

Let $\operatorname{MET}(\mathcal{M})$ be the polyhedron in $\mathbb{R}^{E}$ defined by the inequalities (22) together with $-1 \leq x_{e} \leq 1(e \in E)$. We have $\operatorname{CYC}(\mathcal{M}) \subseteq \operatorname{MET}(\mathcal{M})$. In particular, $\operatorname{CYC}(\mathcal{M})$ is contained in the hyperplane $x_{e}=1$ if $e$ is a coloop of $\mathcal{M}$, and it is contained in the hyperplane $x_{e}-x_{f}=0$ if $e, f$ are coparallel. Thus we may assume without loss of generality that $\mathcal{M}$ has no coloops and no coparallel elements. We will use the following known results.

Lemma 4.3 [2, Corollary 4.21] Let $\mathcal{M}$ be a binary matroid with no $F_{7}^{*}$ minor. The inequality (22) defines a facet of $\mathrm{CYC}(\mathcal{M})$ if and only if $D$ is a chordless cocircuit of $\mathcal{M}$.

Theorem 4.4 $[2$, Theorem 4.22] For a binary matroid $\mathcal{M}, \operatorname{CYC}(\mathcal{M})=\operatorname{MET}(\mathcal{M})$ if and only if $\mathcal{M}$ has no $F_{7}^{*}, R_{10}$ or $\mathcal{M}_{K_{5}}^{*}$ minors.

Recall that $I \mathcal{M}$ is $\mathrm{TH}_{1}$-exact if $\mathrm{CYC}(\mathcal{M})=\mathrm{TH}_{1}(I \mathcal{M})$. 
Lemma 4.5 Assume $\mathcal{M}$ has no $F_{7}^{*}$ minor. If I $\mathcal{M}$ is $\mathrm{TH}_{1}$-exact then $\mathcal{M}$ does not have any chordless cocircuit of length at least five.

Proof Suppose $D=\left\{e_{1}, \ldots, e_{k}\right\}$ is a chordless cocircuit of $\mathcal{M}$ with $k=|D| \geq 5$. By Lemma 4.3, the inequality

$$
x_{e_{1}}-x_{e_{2}}-\cdots-x_{e_{k}} \geq 2-k
$$

defines a facet of $\mathrm{CYC}(\mathcal{M})$. We now use the following claim [2, Lemma 4.17]: For each even subset $F \subseteq D$, there exists a cycle $C \in \mathcal{C}$ for which $C \cap D=F$. Thus we can find three cycles whose intersections with $D$ are respectively $\emptyset,\left\{e_{2}, e_{3}\right\}$ and $\left\{e_{2}, e_{3}, e_{4}, e_{5}\right\}$. Then the linear form $x_{e_{1}}-x_{e_{2}}-\cdots-x_{e_{k}}$ evaluated at each of these three cycles takes the values $2-k, 6-k, 10-k$. In view of Theorem 2.2 we can thus conclude that $I \mathcal{M}$ is not $\mathrm{TH}_{1}$-exact.

Theorem 4.6 Assume $\mathcal{M}$ has no $F_{7}^{*}, R_{10}$ or $\mathcal{M}_{K_{5}}^{*}$ minors. Then I $\mathcal{M}$ is $\mathrm{TH}_{1}$-exact if and only if $\mathcal{M}$ does not have any chordless cocircuit of length at least 5 .

Proof Lemma 4.5 gives the 'only if' part. For the 'if' part, it suffices to verify that, if $D$ is a cocircuit of length at most 4 and $F$ is an odd subset of $D$, then the linear form $\sum_{e \in F} x_{e}-\sum_{e \in D \backslash F} x_{e}$ takes two values when evaluated at cycles of $\mathcal{M}$, and then to apply Theorems 4.4 and 2.2.

Corollary 4.7 The cycle ideal of a graphic matroid $\mathcal{M}_{G}$ is $\mathrm{TH}_{1}$-exact if and only if $G$ has no chordless cut of size at least 5.

Proof Directly from Theorem 4.6 since graphic matroids do not have $F_{7}^{*}, R_{10}$ or $\mathcal{M}_{K_{5}}^{*}$ minors.

Lemma 4.8 If I $\mathcal{M}$ is $\mathrm{TH}_{k}$-exact, then the cycle ideal of any deletion minor of $\mathcal{M}$ is also $\mathrm{TH}_{k}$-exact.

Proof Say $\mathcal{M}^{\prime}=\mathcal{M} \backslash e_{1}$ is a deletion minor of $\mathcal{M}$, where $E=\left\{e_{1}, \ldots, e_{m}\right\}$ and $E^{\prime}=E \backslash\left\{e_{1}\right\}$. Take $\mathbf{x}^{\prime} \in \mathrm{TH}_{k}\left(I \mathcal{M}^{\prime}\right)$; we show that $\mathbf{x}^{\prime} \in \mathrm{CYC}\left(\mathcal{M}^{\prime}\right)$. For this extend $\mathbf{x}^{\prime}$ to $\mathbf{x} \in \mathbb{R}^{E}$ by setting $x_{e_{1}}:=1$. We verify that $\mathbf{x} \in \mathrm{TH}_{k}(I \mathcal{M})$.

For this consider a linear polynomial $f \in \mathbb{R} E$ of the form $f=s+q$ where $s$ is a sos of degree at most $2 k$ and $q \in I \mathcal{M}$. Define the polynomials $f^{\prime}, s^{\prime}, q^{\prime} \in \mathbb{R} E^{\prime}$ by $f^{\prime}\left(x_{e_{2}}, \ldots, x_{e_{m}}\right)=f\left(1, x_{e_{2}}, \ldots, x_{e_{m}}\right)$; similarly for $q^{\prime}, s^{\prime}$. Obviously $s^{\prime}$ is sos with degree at most $2 k$. Since $q$ vanishes on $\left\{\chi^{C}: C \in \mathcal{C}\right\}$, it vanishes on all $\chi^{C}, C \in \mathcal{C}$, with $x_{e_{1}}=1$. This last fact is equivalent to saying that $q^{\prime}$ vanishes on $\left\{\chi^{C}: C \in \mathcal{C}^{\prime}\right\}$. Therefore, $f^{\prime}$ is $k$-sos modulo $I \mathcal{M}^{\prime}$ and so $f^{\prime}\left(\mathbf{x}^{\prime}\right) \geq 0$ as $\mathbf{x}^{\prime} \in \mathrm{TH}_{k}\left(I \mathcal{M}^{\prime}\right)$. In particular, $f(\mathbf{x})=f^{\prime}\left(\mathbf{x}^{\prime}\right) \geq 0$ and $\mathbf{x} \in \mathrm{TH}_{k}(I \mathcal{M})=\operatorname{CYC}(\mathcal{M})$.

Thus $\mathbf{x}$ is a convex combination of \pm 1 -incidence vectors of cycles of $\mathcal{M}$; as $x_{e_{1}}=1$ no cycle in the combination uses $e_{1}$, which thus gives a decomposition of $\mathbf{x}^{\prime}$ as a convex combination of cycles of $\mathcal{M}^{\prime}$.

Remark 4.9 On the other hand, the property of being $\mathrm{TH}_{1}$-exact is not preserved under taking contraction minors. Indeed, every binary matroid can be realized as a contraction minor of some dual binary projective space $\mathcal{P}_{r}^{*}$ (see [10]). Now we observed 
in Example 4.1 that the cycle ideal of $\mathcal{P}_{r}^{*}$ is $\mathrm{TH}_{1}$-exact, while $I \mathcal{M}$ is not always $\mathrm{TH}_{1}$-exact.

See Sect. 5 for examples of cographic matroids whose cycle ideal is $\mathrm{TH}_{2}$-exact while they have a contraction minor whose cycle ideal is not $\mathrm{TH}_{k}$-exact for large $k$ (this is the case for wheels, cf. Corollary 5.10).

We now characterize the $\mathrm{TH}_{1}$-exact cographic matroids. We begin with a lemma relating graph and matroid minors involving $K_{5}$.

Lemma 4.10 The cographic matroid $\mathcal{M}_{G}^{*}$ of a graph $G$ has a $\mathcal{M}_{K_{5}}^{*}$ minor if and only if $K_{5}$ is a contraction minor of $G$.

Proof The 'if part' is obvious since if $K_{5}$ is a contraction minor of $G$, then $\mathcal{M}_{K_{5}}^{*}$ is a deletion minor of $\mathcal{M}_{G}^{*}$. Conversely assume that $\mathcal{M}_{K_{5}}^{*}$ is a minor of $\mathcal{M}_{G}^{*}$. By Whitney's 2-isomorphism theorem (cf. [21]), $K_{5}$ is 2-isomorphic to a minor $H$ of $G$; but then $H$ must be isomorphic to $K_{5}$ as the the only graph 2-isomorphic to $K_{5}$ is $K_{5}$ itself. Hence $K_{5}$ is a minor of $G$, which implies that $K_{5}$ is also a contraction minor of $G$.

Corollary 4.11 The cycle ideal of a cographic matroid $\mathcal{M}_{G}^{*}$ is $\mathrm{TH}_{1}$-exact if and only if $M_{G}^{*}$ has no $M_{K_{5}}^{*}$ minor and no chordless cocircuit of length at least 5 .

Proof Note that $\mathcal{M}_{G}^{*}$ contains no $F_{7}^{*}$ or $R_{10}$ minor. Hence in view of Theorem 4.6, it suffices to show that if $\mathcal{M}_{G}^{*}$ is $\mathrm{TH}_{1}$-exact then $\mathcal{M}_{G}^{*}$ has no $M_{K_{5}}^{*}$ minor. So assume that $\mathcal{M}_{G}^{*}$ is $\mathrm{TH}_{1}$-exact. As $\mathcal{M}_{K_{5}}^{*}$ is not $\mathrm{TH}_{1}$-exact (cf. Example 3.8), Lemma 4.8 implies that $\mathcal{M}_{K_{5}}^{*}$ is not a deletion minor of $\mathcal{M}_{G}^{*}$. Hence $K_{5}$ is not a contraction minor of $G$ which, by Lemma 4.10 , implies that $\mathcal{M}_{K_{5}}^{*}$ is not a minor of $\mathcal{M}_{G}^{*}$.

Reformulating this last result we arrive at a characterization of 'cut-perfect' graphs, answering Problem 8.4 in [18].

Corollary 4.12 The cut ideal of a graph $G$ is $\mathrm{TH}_{1}$-exact if and only if $G$ has no $K_{5}$ minor and no chordless circuit of length at least 5.

In [24, Theorem 3.2], Sullivant obtains the same characterization for compressed cut polytopes; namely he proves that $\operatorname{CUT}(G)$ is compressed if and only if $G$ has no $K_{5}$ minor and no chordless cycles of length at least 5. See [9, Sect. 4] for comments on the connection between compressed polytopes and $\mathrm{TH}_{1}$-exactness.

\section{The theta bodies for cut ideals of circuits}

In this section we determine the exact order $k$ for which the cut ideal $I C_{n}$ of a circuit $C_{n}$ with $n$ edges is $\mathrm{TH}_{k}$-exact. We also obtain some results on graphs whose cut ideal is $\mathrm{TH}_{2}$-exact. We begin with a result determining when the inequalities (22) associated to circuits of $G$ are valid for $\mathrm{TH}_{k}(I G)$.

Theorem 5.1 Let $C$ be a circuit of a graph $G$, let $e \in C$, and let $k$ be an integer such that $4 k \geq|C|$. Then the inequality 


$$
x_{e}-\sum_{f \in C \backslash\{e\}} x_{f} \geq 2-|C|
$$

is valid for $\mathrm{TH}_{k}(I G)$.

The proof uses the following preliminary results. For convenience, for a graph $G=(V, E)$, let $\mathcal{S}_{k}$ denote the set of polynomials $f \in \mathbb{R} E$ that are $k$-sos modulo the cut ideal $I G$.

Lemma 5.2 For a graph $G$, let $F_{1}, F_{2}, F_{3}, F_{4} \subseteq E$ with $\left|F_{i}\right| \leq k$ and such that $F_{1} \Delta F_{2} \Delta F_{3} \Delta F_{4}$ is a cycle of $G$. Then $2+\mathbf{x}^{F_{1}}-\mathbf{x}^{F_{2}}-\mathbf{x}^{F_{3}}-\mathbf{x}^{F_{4}} \in \mathcal{S}_{k}$.

Proof We use the following fact: As $C:=F_{1} \Delta F_{2} \Delta F_{3} \Delta F_{4}$ is a cycle, $1-\mathbf{x}^{C} \in$ $I G$ by Theorem 3.4, and thus $1 \equiv \mathbf{x}^{C} \equiv \mathbf{x}^{F_{1}} \mathbf{x}^{F_{2}} \mathbf{x}^{F_{3}} \mathbf{x}^{F_{4}}$ modulo $I G$. This implies that $\mathbf{x}^{F_{i}} \mathbf{x}^{F_{j}} \equiv \mathbf{x}^{F_{k}} \mathbf{x}^{F_{l}}$ for $\{i, j, k, l\}=\{1,2,3,4\}$. Now, one can easily verify that $\left(2+\mathbf{x}^{F_{1}}-\mathbf{x}^{F_{2}}-\mathbf{x}^{F_{3}}-\mathbf{x}^{F_{4}}\right)^{2} \equiv 4\left(2+\mathbf{x}^{F_{1}}-\mathbf{x}^{F_{2}}-\mathbf{x}^{F_{3}}-\mathbf{x}^{F_{4}}\right)$ modulo $I G$, which gives the result.

Lemma 5.3 For a graph $G$, let $A, B \subseteq E$ with $|A|,|B|,|A \Delta B| \leq k$. Then $1+\mathbf{x}^{A}-$ $\mathbf{x}^{B}-\mathbf{x}^{A \Delta B} \in \mathcal{S}_{k}$.

Proof We have $\left(1+\mathbf{x}^{A}-\mathbf{x}^{B}-\mathbf{x}^{A \Delta B}\right)^{2} \equiv 4+2\left(\mathbf{x}^{A}-\mathbf{x}^{B}-\mathbf{x}^{A \Delta B}\right)+2\left(-\mathbf{x}^{A} \mathbf{x}^{B}-\right.$ $\left.\mathbf{x}^{A} \mathbf{x}^{A \Delta B}+\mathbf{x}^{B} \mathbf{x}^{A \Delta B}\right) \equiv 4\left(1+\mathbf{x}^{A}-\mathbf{x}^{B}-\mathbf{x}^{A \Delta B}\right)$ modulo $I G$.

Lemma 5.4 For a graph $G$, let $F \subseteq E, e \in F$, and $k \geq|F|$. Then:

$$
\begin{aligned}
& \text { (i) } k-1+x_{e}-\sum_{f \in F \backslash\{e\}} x_{f}-\mathbf{x}^{F} \in \mathcal{S}_{k}, \\
& \text { (ii) } k-1-\sum_{f \in F} x_{f}+\mathbf{x}^{F} \in \mathcal{S}_{k} .
\end{aligned}
$$

Proof It suffices to show the result for $k=|F|$. We show (i) using induction on $k \geq 2$. (The proof for (ii) is analogous.) For $k=2, F=\{e, f\}$, we have $1+x_{e}-x_{f}-x_{e} x_{f} \in$ $\mathcal{S}_{2}$ by Lemma 5.3. Consider now $|F|=k \geq 3$ and let $g \in F \backslash\{e\}$. By the induction assumption applied to the set $F \backslash\{g\}$, we have:

$$
k-2+x_{e}-\sum_{f \in F \backslash\{e, g\}} x_{f}-\mathbf{x}^{F \backslash\{g\}} \in \mathcal{S}_{k-1} \subseteq \mathcal{S}_{k} .
$$

Applying Lemma 5.3 to the sets $F \backslash\{g\},\{g\}$ and $F$, we obtain

$$
1+\mathbf{x}^{F \backslash\{g\}}-x_{g}-\mathbf{x}^{F} \in \mathcal{S}_{k}
$$

Summing up the above two relations yield the desired relation (24) (i).

Proof (of Theorem 5.1) Let $C$ be a circuit in $G$ with $|C| \leq 4 k$, i.e. $k \geq m:=\lceil|C| / 4\rceil$. Let $F$ denote the edge set of $C$ and let $e \in F$. We show that the linear polynomial 
$f_{C}:=x_{e}-\sum_{f \in F \backslash\{e\}} x_{f}+|C|-2$ is $k$-sos modulo $I G$. For this we consider a partition of $F$ into four sets $F_{1}, \ldots, F_{4}$ with $\left|F_{i}\right| \leq m \leq k$ for $i=1, \ldots, 4$; say $e \in F_{1}$. Applying Lemma 5.2, we obtain that

$$
2+\mathbf{x}^{F_{1}}-\mathbf{x}^{F_{2}}-\mathbf{x}^{F_{3}}-\mathbf{x}^{F_{4}} \in \mathcal{S}_{k}
$$

Next, applying the condition (24) (i) to $F_{1}$ we obtain

$$
\left|F_{1}\right|-1+x_{e}-\sum_{f \in F_{1} \backslash\{e\}} x_{f}-\mathbf{x}^{F_{1}} \in \mathcal{S}_{k},
$$

and applying the condition (24) (ii) to $F_{i}$ yields

$$
\left|F_{i}\right|-1-\sum_{f \in F_{i}} x_{f}+\mathbf{x}^{F_{i}} \in \mathcal{S}_{k} \forall i=2,3,4 .
$$

Summing up the above relations yields the desired result, namely $f_{C}$ is $k$-sos modulo $I G$ and thus $f_{C} \geq 0$ is valid for $\operatorname{TH}_{k}(I G)$.

Corollary 5.5 For the circuit $C_{n}$ of length $n$, the equality $\mathrm{TH}_{k}\left(I C_{n}\right)=\operatorname{CUT}\left(C_{n}\right)$ holds for $n \leq 4 k$.

Proof Consider the circuit $C_{n}=([n], E)$ with $n \leq 4 k$. By Theorem 4.4, the complete linear description of $\operatorname{CUT}\left(C_{n}\right)$ is provided by the inequalities (i) $\sum_{e \in F} x_{e}-$ $\sum_{e \in E \backslash F} x_{e} \geq 2-n$ where $F$ is any odd subset of $E$, and (ii) $-1 \leq x_{e} \leq 1$ for all $e \in E$. Thus in order to show $\operatorname{TH}_{k}\left(I C_{n}\right)=\operatorname{CUT}\left(C_{n}\right)$, it suffices to show that the inequalities (i), (ii) are all valid for $\mathrm{TH}_{k}\left(I C_{n}\right)$. This is obvious for (ii). Using the well-known switching symmetries of the cut polytope (cf. [2,5]), it suffices to show the desired property for the inequalities (i) with $|F|=1$. But this result has just been shown in Theorem 5.1 .

Lemma 5.6 If $n \geq 4 k+1$, then $\mathrm{TH}_{k}\left(I C_{n}\right)=[-1,1]^{E}$.

Proof In view of Remark 3.5, it suffices to observe that the constraints (14) defining the theta body $\mathrm{TH}_{k}\left(I C_{n}\right)$ reduce to the constraints (14) (i) and (15). Let $\mathcal{F}_{k}$ be the set indexing the combinatorial moment matrices in the definition of $\operatorname{TH}_{k}\left(I C_{n}\right)$, where we can assume that each $F_{i} \in \mathcal{F}_{k}$ has cardinality at most $k$. Now consider a constraint of type (14) (ii). Since $F_{1}, \ldots, F_{4} \in \mathcal{F}_{k}$ have size at most $k$ and $\Delta_{i} F_{i}$ is a cycle of $C_{n}$, this cycle must be the empty set since $\left|\Delta_{i} F_{i}\right| \leq 4 k<n$. Therefore we have a constraint of type (15).

Corollary 5.7 The smallest order $k$ at which $I C_{n}$ is $\mathrm{TH}_{k}$-exact is $k=\lceil n / 4\rceil$.

Proof Directly from Theorem 5.1 and Lemma 5.6.

Remark 5.8 One can verify that the linear form $x_{e}-\sum_{f \in C_{n} \backslash\{e\}} x_{f}$ takes $\lfloor(n+1) / 2\rfloor$ distinct values at the cut vectors of the circuit $C_{n}$. By (3), this permits to conclude that $I C_{n}$ is $\mathrm{TH}_{k}$-exact for $k=\lfloor(n+1) / 2\rfloor-1$. This value is however larger than the order $\lceil n / 4\rceil$ shown in Corollary 5.7 (for $n \geq 6$ ). Thus the reverse implication of (3) does not hold. 
Corollary 5.9 If the graph $G$ has no $K_{5}$ minor and no chordless circuit of length at least 9 , then its cut ideal IG is $\mathrm{TH}_{2}$-exact.

Proof Direct application of Theorems 4.4 and 5.1.

Note that the reverse implication in Corollary 5.9 does not hold. We will see below (in Corollary 5.10) that the cut ideal of a wheel is $\mathrm{TH}_{2}$-exact, but a wheel can contain a chordless circuit of arbitrary length.

While we could characterize the graphs whose cut ideal is $\mathrm{TH}_{1}$-exact, it is an open problem to characterize the graphs whose cut ideal is $\mathrm{TH}_{2}$-exact. We conclude this section with several observations about these graphs.

Corollary 5.10 If the graph $G$ has no $K_{5}$ minor and has diameter at most 2 then its cut ideal IG is $\mathrm{TH}_{2}$-exact.

Proof As $G$ has diameter at most 2, Lemma 3.10 gives the inclusion $\mathrm{TH}_{2}(I G) \subseteq$ $Q_{2}(G)$. It was shown in [15] that if $G$ has no $K_{5}$ minor then $Q_{2}(G)=\operatorname{CUT}(G)$.

A wheel of length $n$ is a graph consisting of a circuit of length $n$ with an additional vertex adjacent to all vertices on the circuit. As wheels have no $K_{5}$ minor and their diameter is 2 , their cut ideal is $\mathrm{TH}_{2}$-exact. Hence, within graphs with no $K_{5}$ minors, the cut ideal is $\mathrm{TH}_{2}$-exact for the following two classes: graphs with diameter at most 2 and graphs with no chordless circuit of size at least 9 . Note that there is no containment between these two classes; e.g. wheels of length $n \geq 9$ have diameter 2 but contain a circuit of length $n$, and $C_{8}$ has diameter larger than 2 .

The following further graphs have a $\mathrm{TH}_{2}$-exact cut ideal: $K_{5}, K_{6}, K_{7}$ (and probably $K_{8}$ too, as conjectured in [15]). Finally, if the cut ideal of a graph $G$ is $\mathrm{TH}_{2}$-exact, then the same holds for the cut ideal of any contraction minor $H$ of $G$; in particular, $C_{9}$ is not a contraction minor of $G$.

Open Access This article is distributed under the terms of the Creative Commons Attribution Noncommercial License which permits any noncommercial use, distribution, and reproduction in any medium, provided the original author(s) and source are credited.

\section{References}

1. Barahona, F.: The max-cut problem on graphs not contractible to $K_{5}$. Oper. Res. Lett. 2, 107-111 (1983)

2. Barahona, F., Grötschel, M.: On the cycle polytope of a binary matroid. J. Combinatorial Theory Ser. B 40(1), 40-62 (1986)

3. Bochnak, J., Coste, M., Roy, M.-F.: Real Algebraic Geometry. Springer, Berlin (1998)

4. Cox, D.A., Little, J.B., O’Shea, D.B.: Ideals, Varieties and Algorithms: An Introduction to Computational Algebraic Geometry and Commutative Algebra. Springer, Berlin (2005)

5. Deza, M.M., Laurent, M.: Geometry of Cuts and Metrics. Springer, Berlin (1997)

6. Edmonds, J., Johnson, E.L.: Matching, Euler tours and the Chinese postman. Math. Program. 5, 88-124 (1973)

7. Garey, M.R., Johnson, D.S.: Computers and Intractability: A Guide to the Theory of NP-Completeness. W. H. Freeman \& Company, Publishers, San Francisco (1979)

8. Goemans, M.X., Williamson, D.: Improved approximation algorithms for maximum cuts and satisfiability problems using semidefinite programming. J. ACM 42, 1115-1145 (1995)

9. Gouveia, J., Parrilo, P.A., Thomas, R.: Theta bodies for polynomial ideals, preprint. arXiv:0809.3480 (2008) 
10. Grötschel, M., Truemper, K.: Master polytopes for cycles of binary matroids. Linear Algebra Appl. 114/114, 523-540 (1989)

11. Grötschel, M., Truemper, K.: Decomposition and optimization over cycles in binary matroids. J. Combinatotorial Theory B 46(3), 306-337 (1989)

12. Lasserre, J.B.: An explicit exact SDP relaxation for nonlinear 0-1 programs. In: Aardal, K., Gerards, A.M.H. (eds.) Lecture Notes in Computer Science, vol. 2081, pp. 293-303 (2001)

13. Laurent, M.: A comparison of the Sherali-Adams, Lovász-Schrijver and Lasserre relaxations for 0-1 programming. Math. Oper. Res. 28(3), 470-496 (2003)

14. Laurent, M.: Lower bound for the number of iterations in semidefinite relaxations for the cut polytope. Math. Oper. Res. 28(4), 871-883 (2003)

15. Laurent, M.: Semidefinite relaxations for max-cut. In: The sharpest cut, MPS/SIAM Ser. Optim., pp. 257-290. SIAM, Philadelphia, PA (2004)

16. Laurent, M.: Semidefinite representations for finite varieties. Math. Program. 109, 1-26 (2007)

17. Laurent, M., Rendl, F. : Semidefinite programming and integer programming. In: Aardal, K., Nemhauser, G., Weismantel, R. (eds.) Handbook on Discrete Optimization, pp. 393-514. Elsevier B.V., Amsterdam (2005)

18. Lovász, L.: On the Shannon capacity of a graph. IEEE Trans. Inf. Theory IT-25, 1-7 (1979)

19. Lovász, L.: Semidefinite programs and combinatorial optimization. In: Recent advances in algorithms and combinatorics, volume 11 of CMS Books Math./Ouvrages Math. SMC, pp. 137-194. Springer, New York (2003)

20. Lovász, L., Schrijver, A.: Cones of matrices and set-functions and 0-1 optimization. SIAM J. Optim. 1, 166-190 (1991)

21. Oxley, J.G.: Matroid Theory. Oxford University Press, Oxford (1992)

22. Parrilo, P.A.: Semidefinite programming relaxations for semialgebraic problems. Math. Prog. 96(2, Ser. B), 293-320 (2003)

23. Sherali, H.D., Adams, W.P.: A hierarchy of relaxations between the continuous and convex hull representations for zero-one programming problems. SIAM J. Discr. Math. 3, 411-430 (1990)

24. Sullivant, S.: Compressed polytopes and statistical disclosure limitation. Tohoku Math. J. (2) 58(3), 433-445 (2006)

25. Wiegele, A.: Nonlinear optimization techniques applied to combinatorial optimization problems. $\mathrm{PhD}$ thesis. Alpen-Adria-Universität Klagenfurt (2006) 